\title{
Firefighter Turnout Gear Durability Study-Evaluation of Visibility Trim
}

\author{
Deena Cotterill* and Elizabeth P. Easter, University of Kentucky, \\ Lexington, USA
}

Received: 24 June 2010/Accepted: 27 September 2010

\begin{abstract}
The high visibility trim requirements for NFPA 1971 (2007 ed.) turnout gear are intended to provide the user conspicuity enhancements in both daytime and nighttime environments. This study evaluated the reflectance, color and fluorescence properties of high visibility trim on firefighters used turnout gear. Forty-four used garments, grouped in age categories from 2 to 10 years (retired), were visually evaluated according to the Section 4 of the NFPA 1851-Advanced Inspection Checklist. Advanced inspection identified damaged trim on 21 of the 46 garments. The damaged areas, located primarily at the cuffs and lower front hem, were not as reflective but passed the subjective flashlight test. A data sampling plan for retroreflective values was constructed for the coats and pants and the coefficients of retroreflection were collected to provide a measure of the nighttime conspicuity. To characterize the fluorescent (daytime) properties of the trim on the used garments the values for fluorescence and color box coordinates were measured. The measured coefficient of retroreflection $\left(\mathrm{R}_{\mathrm{A}}, \mathrm{cd} / \mathrm{lux} / \mathrm{m}^{2}\right)$ for all garments (average of all values in the sampling plan) was greater than $3 \times$ the minimum NFPA 1851 requirement. Therefore, all used gear provided substantial nighttime conspicuity enhancement including the retired gear.
\end{abstract}

Keywords: Visibility trim, Turnout gear, NFPA 1851, NFPA 1971, Firefighter

\section{Introduction}

\subsection{Background of Turnout Gear High Visibility Trim and Firefighter Environments}

Structural firefighting is an intense, strenuous and hazardous occupation that exposes firefighters to a wide variety of challenging environmental conditions including extreme temperatures, hazardous chemicals and blood borne pathogens. In 2007, over 80,000 firefighters were injured and 103 were killed while responding to emergencies or fighting fires according to a National Fire Protection Association report [1]. Protective clothing, also known as personal protective equipment or PPE, is a fire fighter's first line of defense to minimize the risk of injury in these hazardous environments. The structural firefighter's protective ensemble consists of a coat, pants, boots, helmet, gloves, hoods and any other equipment a firefighter wears when responding to an emergency. The coat and pants of the protective ensemble, commonly referred to as turnout gear, provides primary protection to the fire fighter. They are donned

\footnotetext{
* Correspondence should be addressed to: Deena Cotterill, E-mail: text.lab.uky@gmail.com
} 
by the fire fighter as outerwear before entering an environment; worn over other clothing and removed after exiting the hazardous environment.

To protect the firefighter from the wide range of harsh conditions, the coat and pants are composed of three primary component elements: an outer shell, a moisture barrier, and a thermal liner, as specified in the standard NFPA 1971 (2007 ed.), Standard on Protective Ensembles for Structural Firefighting and Proximity Firefighting [2]. Supplementing these primary elements are other functional construction features, one of which is high visibility trim. The trim functions by enhancing visual conspicuity, or a wearer's ability to be noticed by an observer, during daytime, nighttime, and dawn/dusk periods. The trim is an important element of the turnout gear, as firefighters rely on their visual conspicuity during firefighting and in their many non-firefighting activities, for location and identification of themselves and coworkers, and as part of a strategy to reduce risks from being hit by hazards such as vehicles. Worker injuries and fatalities of these types are listed in accident statistical documents under the category of struck-by incidents. Photometric properties, amounts, and design/placement of these materials on high visibility garments are specified in garment standards, in this case the NFPA 1971 ensemble standard. The high visibility requirements for NFPA 1971 turnout gear have been largely stable for the last 10 years of standards revisions.

High visibility trim consists of retroreflective and fluorescent conspicuity enhancing materials. Retroreflective materials improve nighttime conspicuity primarily by increasing luminance contrast against a background, with significant contributions from spatial frequency (size and pattern) and motion cues. Retroreflective material, which returns (reflects) light for example from a vehicle headlight approximately back to its source and on a roughly co-linear path to the eyes of an observer (the driver), is primarily used to enhance nighttime conspicuity. The trim consists of millions of small reflective elements. When a light source like a flashlight beam hits the trim it is reflected back to the source making the trim highly visible in poor visibility situations ("How $3 \mathrm{M}^{\mathrm{TM}}$ Scotchlite ${ }^{\mathrm{TM}}$ Reflective Material Works," n.d. [3]). Most new high visibility material starts at approximately $500 \mathrm{R}_{\mathrm{A}}$ (coefficient of retroreflection, cd/lux $/ \mathrm{m}^{2}$ ) or higher, well above the $100 \mathrm{R}_{\mathrm{A}}$ requirement in the NFPA 1971 standard.

Fluorescent enhancements improve daytime, dawn/dusk, and partial light conspicuity [4]. Fluorescent materials, which contain pigments or dyes that convert some UV light not visible to the naked eye in the environment into additional bright visible light, improve conspicuity by enhancing color contrast, luminance contrast, spatial frequency, and motion against a background or competing scene distracters. The NFPA 1971 standard specifies a prescribed placement pattern and minimum material dimensions, thus defining both a minimum amount of material required and creating uniform effective geometry for a recognizable look among users. Several variations of NFPA 1971 compliant high visibility materials exist, however, this study examined only one predominant type in two color variations in its measurement portion. Besides the NFPA 1971 specification, other example standards for high visibility garments in use globally by other workers and sometimes by firefighters are ANSI/ISEA 107, ANSI/ISEA 207, CSA Z96, EN 471, and EN469 (European structural firefighting standard) [5-9]. A recently published 
study compared the relative effectiveness of some of these types of high visibility garments in the U.S. (NFPA 1971, ANSI 107, ANSI 207) at creating conspicuity for their wearers, and concluded that they perform nearly identically in both daytime and nighttime simulated emergency scene environments, Tuttle [10].

The high visibility patterns present in NFPA 1971 turnout gear are notably effective because they distribute material widely across the torso and limbs, to capture both the motion and shape of the wearer. Material at the very end of the limbs and major joints has been shown to optimize pedestrian conspicuity, possibly by providing strong sympathetic visual motion clues to an observer that the wearer is human $[11,12]$. This type of pattern is often described as "biomotion" and has been found to be highly efficient at creating conspicuity for its wearer, as compared to patterns which confine high visibility materials to the torso only. The minimum performance for new retroreflective materials in the NFPA standard is $100 \mathrm{R}_{\mathrm{A}}$. Scientific evidence indicates material performance values near this number, and probably at lower values, when present in a standardized bright-banded pattern in a design-compliant ensemble, represent effective functional minimums for in-use retroreflective material used for conspicuity enhancement in complex scenes [13]. For the purpose of this analysis, $100 \mathrm{R}_{\mathrm{A}}$ is considered the performance point of reference for in-use material.

When firefighters work in extreme environments of high heat, flame, potential explosions, thermal flashover, chemical exposures, and rough physical exposures, the conditions can affect the materials of the turnout gear. These environments may degrade the materials of the firefighter's personal protective equipment (PPE), resulting in reduced garment performance and possible increased risk of job-related injuries. Degradation of garment materials in this kind of use may be inevitable, yet firefighters need assurance their gear will continue to provide effective protection over the course of its useful service life. Ideally, the wearer or responsible issuer of the equipment will be able to assume that throughout use, materials can continue to perform effectively over the mandated lifetime, or that it will be possible to readily inspect and identify PPE which may be degraded beyond an acceptable minimum level, so the gear may be marked for timely repair or replacement. The purpose of the parent study, Post-Use Evaluation of Fire Fighter's Turnout Gear, by Cotterill [14] on in-use firefighter coats and pants, was to evaluate the performance of used turnout gear. This research project evaluated firefighter protective clothing performance against the requirements of the NFPA 1851 to 2008 standard, Standard on the Selection, Care, and Maintenance of Structural Fire Fighting Protective Ensembles [15], over the 10 year maximum lifetime. This report addresses the specific performance of the visibility trim on used turnout gear during this timeframe.

\section{Methodology}

\subsection{Sample}

Forty-four garments (23 coats and 21 pants) were collected from medium and large fire departments. They were divided into three categories according to age, 
from 2 to 3 years (20 garments), 5 to 7 years (14 garments) and 9 to 10 years or retired (10 garments, reported as separate in some data analyses). For safety reasons, all garments were cleaned prior to evaluating the trim. The high visibility trim on all 44 garments was subjected to the Advanced Inspection criteria of NFPA 1851. Performance of retroreflectivity and fluorescence was measured at the 3M Occupational Health and Environmental Safety Tech Service laboratory facility in Saint Paul, Minnesota.

\subsection{Evaluations and Tests}

All used turnout gear were subjected to the Advanced Inspection criteria of the NFPA 1851 standard. The basic criteria for high visibility materials inspection includes visually examining the coat and trousers for damaged or missing reflective trim. NFPA 1851 advanced inspection requires that all separable layers of the garment elements including the reflective trim be individually inspected for integrity, attachment to garment, reflectivity or damage (National Fire Protection Association, 2007).

Advanced Inspections included a subjective evaluation of the retroreflective properties by following the procedures of the flashlight test of the 2008 edition of NFPA 1851 section A.6.3.5.1 (9). A test garment and a piece of new trim were placed $40 \mathrm{ft}(12 \mathrm{~m})$ from the evaluators, in a dark room without outside light interference. Materials on the front and the back of each garment were evaluated separately using two evaluators, but the ratings were combined for one rating per garment. Evaluators held a bright, focused flashlight at eye level, next to their temple and aimed the light beam at the samples. Evaluators compared the garments to the new sample and rated the garment "pass" or "fail". An area with discernibly reduced reflectivity, i.e., not as reflective as the rest of the garment, were noted as damaged trim.

To evaluate the performance of high visibility trim quantitatively the retroreflective and fluorescent properties of the trim were tested. The Coefficients of Retroreflection $\left(\mathrm{R}_{\mathrm{A}}\right)$ were collected using ASTM method E809-Standard Practice for Measuring Photometric Characteristics of Retroreflectors [16] on the $3 \mathrm{M}$ instrument Retrophotometer RM-2 with $0.2^{\circ}$ observation angle and $5^{\circ}$ entrance angle and a $1 / 2^{\prime \prime}(1.27 \mathrm{~cm})$ aperture after calibration to an appropriate level retroreflector standard plaque. It is reported in $\mathrm{Cd} / \mathrm{lux} / \mathrm{m}^{2}$ (ASTM, 2008). The retroreflective values of the trim were sampled in 46 locations on each coat and 12 locations on each pair of pants as shown in Figure 1. All together a total of 58 data points were measured on the coats and pants as an attempt to give a holistic representation of the performance of the majority of the garment. One coat did not have a second set of trim on the upper sleeve, reducing the number of testing locations for that coat.

The daytime conspicuity was evaluated by measuring the fluorescence of the yellow or orange sections of the trim on a spectrophotometer to evaluate the values for fluorescence luminance (Y) and color box coordinates (x, y), thus characterizing the fluorescent and color properties of the high visibility material, as defined in NFPA 1971 (2007 ed.). In-use values were measured per ASTM E-1164 


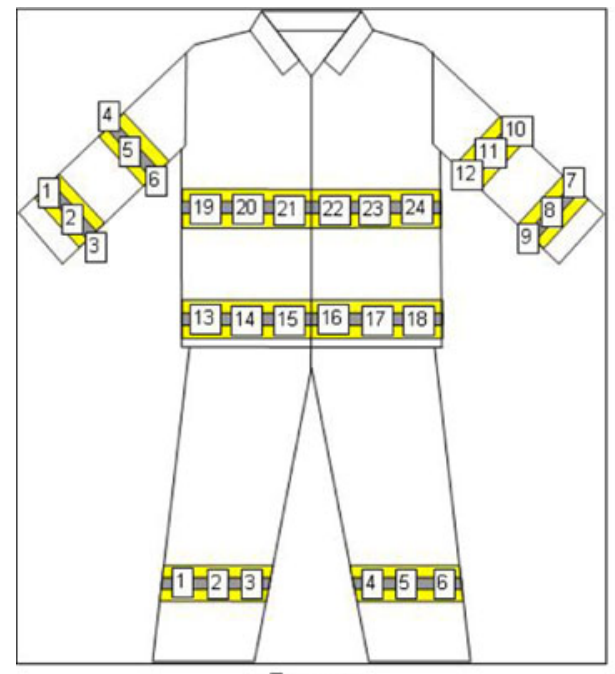

Front

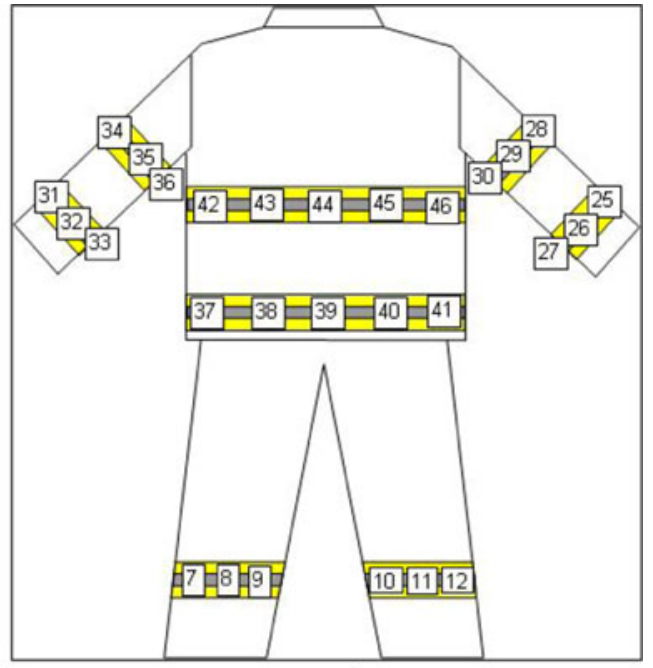

Back

Figure 1. Diagram of test locations for coefficients of retroreflection.

using a HunterLab ColorFlex 45/0 spectrophotometer under a D65 Standard Illuminant with a $2^{\circ}$ observer.

\section{Data Analysis}

Numerical analysis was used with corresponding charts and graphs to communicate the results of this study. Specific techniques include descriptive statistics, a two sample $t$-test, and interval plots by group for continuous data. Attribute agreement analysis was employed to validate field tests versus laboratory tests where applicable. Six Sigma benchmarks $(P<0.0001)$ were used to determine if an agreement was acceptable. In order for there to be an acceptable agreement there must be an overall Kappa of $0.9(P<0.0001)$. Bar graphs are used to communicate the details of the data. Levels of significance were calculated using Minitab ${ }^{\circledR}$ statistical software.

\section{Results}

\subsection{Subjective Evaluation-Advance Inspection}

Results of the Advance Inspection showed that overall the cleanliness of the trim was good. The evaluation of trim attachment (stitching) found the trim on eight garments was not securely attached and the assessment of damaged trim showed that 20 garments had damaged areas of one inch or greater (Table 1). Trim on all 44 garments passed the flashlight assessment test, however, four garments had small, less reflective areas due to heavy soiling. Even though 20 garments had at least one damaged area the trim remained reflective enough to pass the flashlight 


\section{Table 1}

Advanced Inspection of Trim

\begin{tabular}{lccc}
\hline & Securely attached & Damage 1" of greater & Flashlight test \\
\hline All garments & 36-Yes & 14-Yes & 46-Pass \\
2-3 years & 7-No & 5-Yes & 0-Fail \\
5-7 years & 1-No & 4-Yes & 0-Fail \\
Retired garments & 0-No & 5-Yes & 0-Fail \\
\hline
\end{tabular}

test. Two of the garments that received advanced inspection were not measured in the laboratory because they were a different form of trim that would not be statistically compatible. Trim on two garments exhibited some loss of reflectivity on the lettering on the back of the coat. Inspection of these also showed heavy soiling of the letters. The reflectivity of the lettering on the back of the coat is not a requirement and not counted as part of the reflectivity readings.

\subsection{Instrumental Evaluation-Retroreflectivity}

The coefficient of retroreflectivity, $\mathrm{R}_{\mathrm{A}}$, on most new high visibility material starts at approximately $500 \mathrm{R}_{\mathrm{A}}$ or higher, well above the $100 \mathrm{R}_{\mathrm{A}}$ requirement in the NFPA 1971 standard. It has also been shown previously by Sayer that the $\mathrm{R}_{\mathrm{A}}$ of the high visibility material on a standard compliant garment may fall to 100 $\mathrm{Cd} / \mathrm{lux} / \mathrm{m}^{2}$ or lower with minimal impact on performance as represented by detection distance in a visual search task, Sayer [13]. The effectiveness of the high visibility materials on the coats and pants at creating nighttime conspicuity enhancement has been shown within certain design parameters to be jointly a function of the total amount of light returned to an observer by the ensemble and the placement geometry of the retroreflecting materials (Linzie, Anderson, Casner, et al., in preparation). Together, these create a justifiable rationale that calculating the average $\mathrm{R}_{\mathrm{A}}$ value over a substantial number of evenly distributed sample spots representing the entirety of the garment is a useful way to estimate the degree of degradation that the garment has experienced, as long as in no cases has all of the degradation occurred in one particular viewing quadrant, which would completely obscure the wearer to an observer from that angle.

The performance of the coats averaged $320 \mathrm{Cd} / \mathrm{lux} / \mathrm{m}^{2}$ in the 2 to 3 age category; $378 \mathrm{Cd} / \mathrm{lux} / \mathrm{m}^{2}$ in the 5 to 7 age category; $308 \mathrm{Cd} / \mathrm{lux} / \mathrm{m}^{2}$ in the 9 to 10 age group and $325 \mathrm{Cd} / \mathrm{lux} / \mathrm{m}^{2}$ for retired gear. The average coefficient of retroreflection $\left(\mathrm{R}_{\mathrm{A}}\right)$ value of the 23 fire coats was $337 \mathrm{Cd} / \mathrm{lux} / \mathrm{m}^{2}$, and $310 \mathrm{Cd} / \mathrm{lux} / \mathrm{m}^{2}$ for the pants. Performance of the pants in the 2 to 3 -age category averaged $308 \mathrm{Cd} / \mathrm{lux} / \mathrm{m}^{2} ; 303$ $\mathrm{Cd} / \mathrm{lux} / \mathrm{m}^{2}$ in the 5 to 7 age category; $306 \mathrm{Cd} / \mathrm{lux} / \mathrm{m}^{2}$ in the 9 to 10 age group and $233 \mathrm{Cd} / \mathrm{lux} / \mathrm{m}^{2}$ for retired gear. The average coefficient of retroreflection $\left(\mathrm{R}_{\mathrm{A}}\right)$ value of the 23 fire coats was $337 \mathrm{Cd} / \mathrm{lux} / \mathrm{m}^{2}$, and $310 \mathrm{Cd} / \mathrm{lux} / \mathrm{m}^{2}$ for the pants. Garment averages in all age categories as a whole far exceed the NFPA 1971 minimum requirement for new trim, $100 \mathrm{R}_{\mathrm{A}}$. A breakdown of the average retroreflectance by age and by garment type can be found in Figures 2 and 3. 


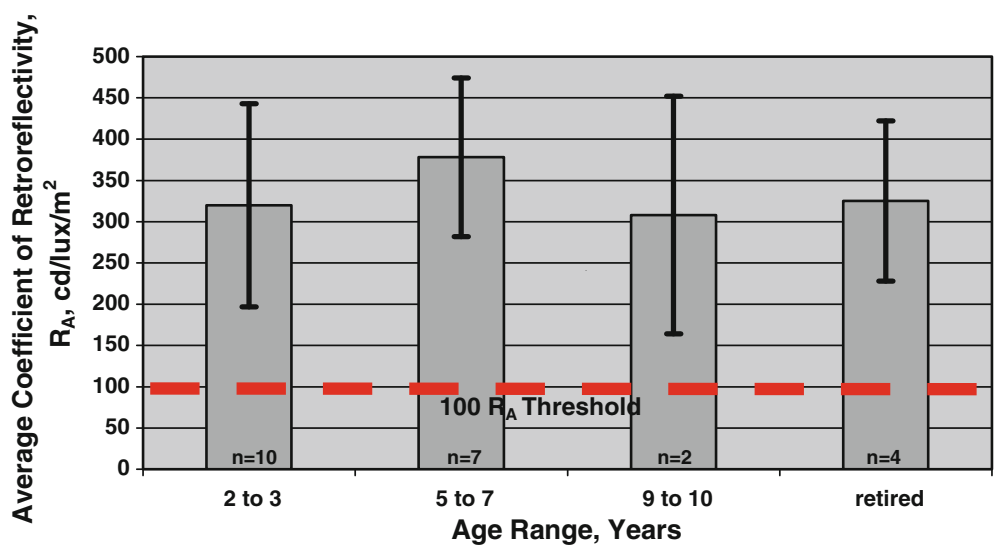

Figure 2. Average coat coefficient of retroreflectivity $\left(R_{A}\right)$ by age category, 46 sample spots per garment, error bars represent \pm one standard deviation.

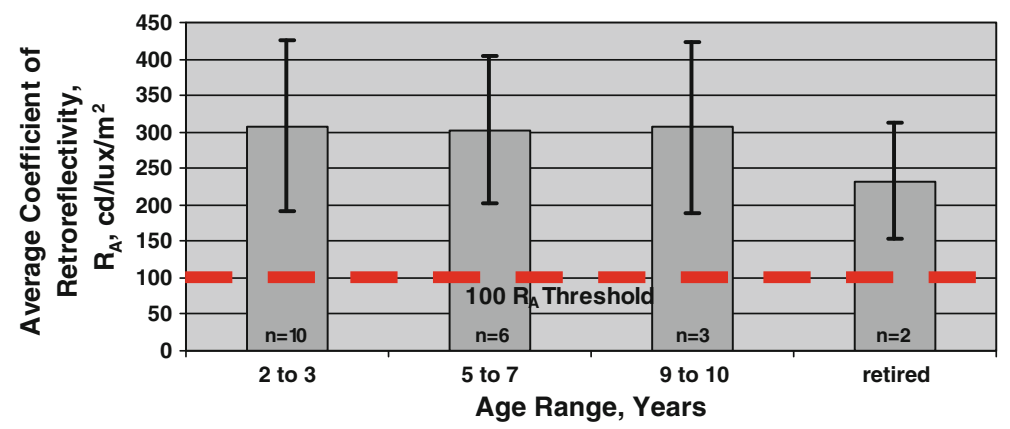

Figure 3. Average pant coefficient of retroreflectivity $\left(R_{A}\right)$ by age cafegory, 12 sample spots per garment, error bars represent \pm one standard deviation.

The data showed a normal bell curve indicating both natural and measurement variability, some of which may be the result of sampling bias. First, the age category at 2-3 years included some garments with a more recent variant of trim material with significantly lower initial $\mathrm{R}_{\mathrm{A}}$ and potentially different wear properties. This variant, which lowered the aggregate average value of the 2- to 3-year garments, was not present in the other age categories, and may account for the lower apparent average of the 2- to 3-year material compared to the older samples. Secondly, the garments which were collected for testing were specifically selected for free replacement, and in the absence of a blind random selection process from departments' inventories, it is reasonable to conclude that the person selecting fire suits for free replacement would naturally remove those which were most worn, i.e., the human selector would tend to a bias toward a worst wear 


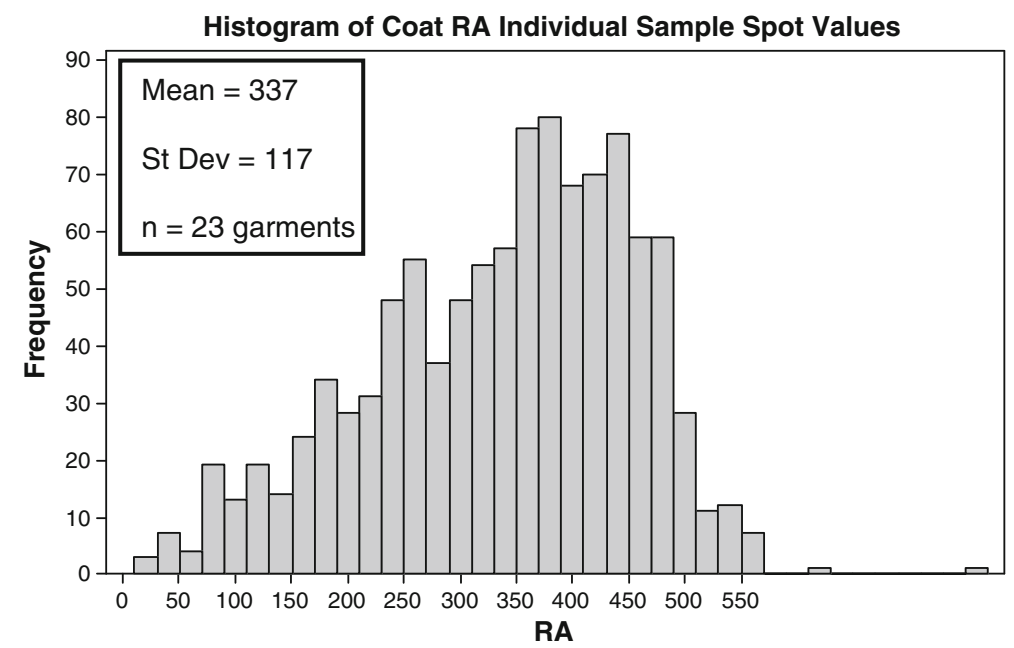

Figure 4. Histogram of coat $R_{A}$ individual sample spot values.

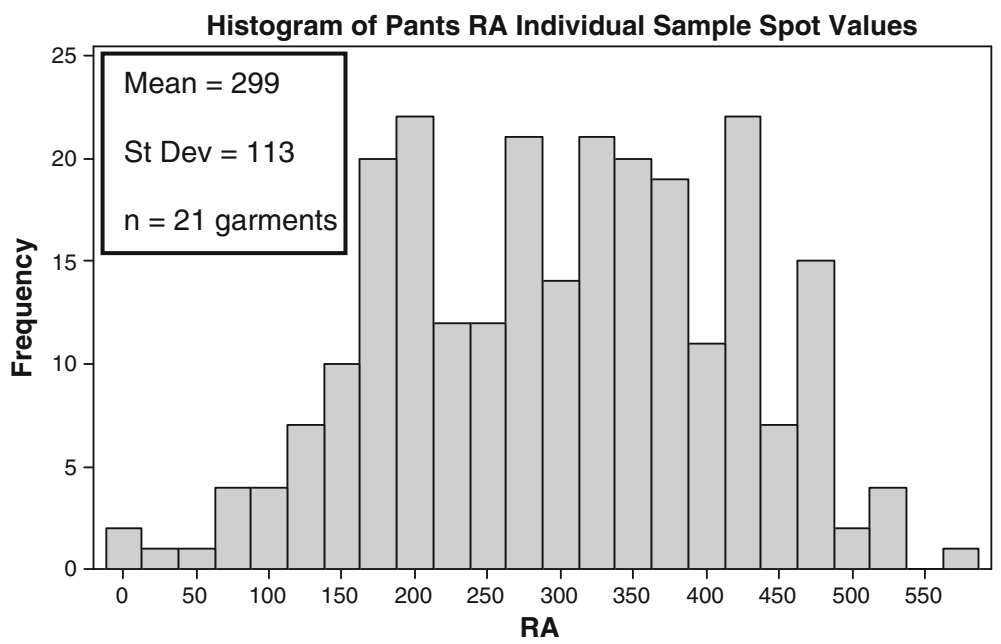

Figure 5. Histogram of pant $R_{A}$ individual sample spot values.

scenario. In the data set the majority of low photometric readings came from a relatively few badly worn or damaged garments in the population, for example fully one-third of the $3.8 \%$ of coat spot readings below $100 \mathrm{R}_{\mathrm{A}}$ came from a single badly worn yet relatively young garment (in the 2- to 3-year category) out of the population of 23 turnout coats. Histograms of all collected $R_{A}$ data points for the turnout coat and pants sample populations are presented in Figures 4 and 5. Further, inspection indicates that the most worn regions of the high visibility pattern were indicated (numerically) to be the left and right coat sleeve cuffs, and the 
lower front stripe center near the hem, spots 1 to 3,7 to 9,14 to 17,25 to 27 , and 31 to 33 , Figure 1.

\subsection{Instrumental Evaluation-Fluorescence}

Measured detection distances in visual search tasks for pedestrians wearing standardized high visibility garments in natural or simulated complex scenes are almost always shorter at night than during the day, suggesting that enhancing nighttime visibility of pedestrians in dark environments is the more challenging task of high visibility materials and is more critical to wearer safety. For that reason, greater effort was applied to evaluating nighttime (retroreflective) performance of the in-use garments in this study. Examples of daytime and nighttime detection distances quantified in visual search tasks in simulated scenes can be seen in the published work for example of Sayer [13, 17], Luoma [18], and Tyrrell [11].

To better understand the durability issues of materials providing daytime fluorescence properties in high visibility garments, one may consider that in a generic supplemental high visibility garment such as a basic ANSI 107 Class 2 highway vest, the fading, or loss of color, in the background fabric from sun exposure can be a typical common failure mechanism that prompts the retirement of the garment. The United States Federal Highway Administration has concluded from its own studies that the expected lifetime of such a garment worn outside for everyday work is approximately 6 months, and approximately 3 years for a garment not worn every day (Federal Register [19]). Even though overall cleanliness of the trim was found to be good, because of the unusual length and harshness of environmental exposure of up to 10 years in the case of firefighter turnout gear, it is important to investigate the longevity of the fluorescent properties of the high visibility materials.

This examination found that predicted repeated exposures to heat, flame, sunlight, and care procedures associated with firefighting do not appear by themselves to significantly degrade the daytime properties of high visibility materials in this study. Evidence of this is that some garments in the 5- to 7-year and 9- to 10-year categories had original trim with retroreflection $\left(\mathrm{R}_{\mathrm{A}}\right)$, color box, and cap $\mathrm{Y}$ values equivalent or nearly equivalent to new requirements. Of greater interest, therefore, are other types of wear that would be expected to impact fluorescent properties, specifically staining and sources of discoloration such as scorching. These kinds of degrading wear can expectedly shift the color box and luminance (cap Y) properties of the fluorescent materials. Color box coordinate measurement is an indication of the color fading or shifting from its initial value, while cap Y quantifies the capability of the fluorescent dyes or pigments to convert ultraviolet wavelengths to visible light.

To evaluate the impact of these effects, measurements of color and luminance properties were deliberately sampled from garments selected for observably high wear on the lower stripe on the front of the coat, roughly corresponding to sample areas 13 to 18, Figure 1, representing instances where staining and discoloration were plainly evident. This location, corresponding to a contact point for tools or 


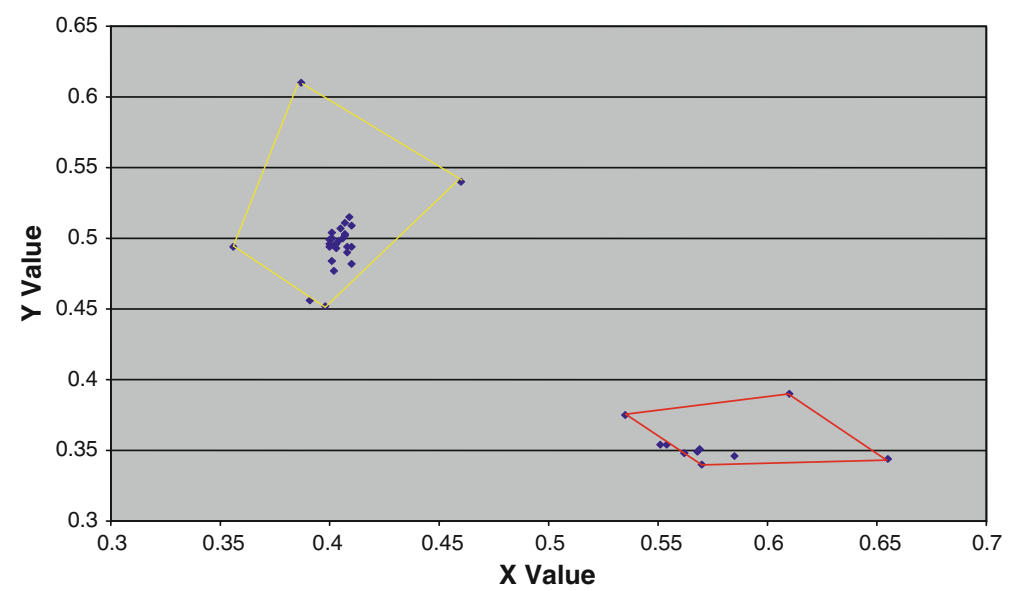

Figure 6. Color box values for the fluorescent trim on the coats.

objects transported manually or gripped by the firefighter, was often visibility stained or worn worse than other areas of the garments, and therefore may be considered something of a worse case.

Evaluating the daytime conspicuity, the fluorescent portion of the trim showed that most samples fell within the ANSI color box values. As expected, the presence of staining or visual discoloration was often an indication of a shift in color box values for samples in both fluorescent lime yellow and fluorescent orange-red, Figure 6. In some samples, the stains moved color coordinates slightly beyond the boundaries of the defined color space. Likewise, cap Y values were also predictably shifted lower where discoloration or staining was evident.

It should be noted that the areas with heavy soiling and discoloration fell on the parameter or just outside of the color box (x,y); this can be seen in Figure 6 . The areas of heavy soiling and discoloration typically observed on the sample garments were around the wrists where the firefighter held and carried items and on the lower front hem of the jacket.

\subsection{Care and Maintenance Considerations}

The levels of heat, flame, smoke, chemicals, sunlight exposure, and care procedures that the high visibility material on the turnout garments in this study experienced in a lifetime of structural firefighting do not appear by themselves to have caused degradation that would significantly compromise its performance, as even some garments in the older categories retained photometric properties nearly equivalent to new. The average $R_{A}$ value of every garment evaluated was well above the NFPA minimum, and they would return significant reflected luminance to enhance the nighttime conspicuity of the wearer.

Most degradation occurred in specific areas. The most-worn areas were the arm and pant cuffs and the front lower edge of the jacket, where one may expect the garment to experience elevated abrasion, rubbing, staining, discoloration, or wear leading to loss of retroreflectivity and fluorescent properties. Of the 40 total spots 
measuring below $100 \mathrm{R}_{\mathrm{A}}$ on the jackets, a disproportionate $83 \%$ of those spots were from the sleeve cuffs or lower front hem areas. These areas comprise $35 \%$ of the sample spots. Approximately one-third of the low-measuring spots were from a single garment, while the remaining spots were distributed over nine coats. Four of those coats had only one spot measuring below 100, with an average value of 77. Interestingly, only one jacket had a single spot that measured below 100 on the back, suggesting that SCBA use did not have a strong wearing effect on the high visibility materials on this population of garments.

An effective care and maintenance program for high visibility trim materials would emphasize a visual inspection on the cuffs and front hem of the jacket. Areas which appear heavily abraded, stained, or discolored would be good candidates first for material-compatible spot cleaning, and secondly for repair when the garment goes in for maintenance work. Evidence of the effectiveness of an inspect and repair approach was present on at least one garment in the sample population, which had approximately $40 \%$ of the trim replaced on the pants and sleeves at some point in its 9- to 10-year service life.

\section{Conclusions}

The high visibility material evaluated in this study maintained its performance well throughout the useful life of the garments, with the average coefficient of retroreflection across the sample population measuring several times higher than minimum requirements. Though approximately $4 \%$ of the measured spots were below $100 \mathrm{R}_{\mathrm{A}}$, even the most worn garment, which accounted for approximately one-third of all the low values, averaged well above minimum and would be expected to return a significant amount of light to provide useful and effective nighttime conspicuity enhancement based on current scientific understanding.

Fluorescent properties also held up well over the 10-year lifetime, with some garments in the 5- to 7-year and 9- to 10-year categories having color box coordinate and cap $\mathrm{Y}$ values nearly equivalent to new, indicating that given reasonable cleaning and care, the kinds of repeated exposures associated with structural firefighting by themselves are not necessarily problematic for the high visibility materials over this timeframe. Where staining or other forms of heavy wear or discoloration were evident by visual inspection, this was a good indicator that cap $\mathrm{Y}$ or color box coordinates may have shifted. These areas should be noted for further inspection and possible replacement when the garment receives maintenance or repair.

The high visibility component of the structural firefighting coats and pants proved quite robust based on this population of sample garments. While every garment examined from age 2 years through retirement would be expected to provide useful and effective conspicuity enhancement despite some areas showing significant wear, a best practice would be to perform periodic visual inspection for evidence of significant wear and discoloration to indicate where repairs would be efficacious to maintain the highest possible performance of the garment through its useful lifetime. 
Finally, this study compared the flashlight test, a subjective test conducted by a firefighter in the field and specified in the inspection routine in the NFPA 1851 standard, to the instrumental measurement of photometric properties responsible for enhancing conspicuity. The instrumentally measured retroreflectivity test results agreed with the flashlight test in that all of the garments passed. The instrumental reflectivity showed a few locations had weakened in intensity, but all garments passed the NFPA minimal requirement of $100 \mathrm{R}_{\mathrm{A}}$. The trim's fluorescence performance, while not tested in this study as thoroughly as the retroreflective properties, appeared to meet or exceed the minimum requirements for all garments as a whole. Some of the 5- to 7-year and 9- to 10-year categories had readings close to new trim, meaning the fluorescence properties of the trim performed well for the useful life of the garment.

The results confirm the flashlight test specified in NFPA 1851 should enable the firefighter to effectively evaluate trim reflectance on their turnout gear. Even though all garments passed, there were retroreflective areas noted to be more prone to wear, thus the flashlight test would enable the inspector to view and identify weak areas that might need repair in the future.

\section{Acknowledgments}

We would like to thank Ken Hanzalik and Tim Gardner from 3M Occupational Health and Environmental Safety Division for help in testing garments and support of this project. We would also like to thank Richard Young of DuPont, Tricia Hock of Fire Dex, Patricia Freeman of Globe Manufacturing and Karen Lehtonen of Lion Apparel for their support of this project.

\section{References}

1. National Fire Protection Association (2009) NFPA :: Research :: Fire statistics :: The U.S. fire service. http://www.nfpa.org/categoryList.asp?categoryID $=955 \& U R L=$ Research/ Fire\%20statistics/The\%20U.S.\%20fire\%20service. Accessed 6 Jun 2009

2. National Fire Protection Association (2006) NFPA 1971 standard on protective ensembles for structural fire fighting and proximity fire fighting. NFPA, Quincy, MA, p 126

3. How $3 \mathrm{M}^{\mathrm{TM}}$ Scotchlite ${ }^{\mathrm{TM}}$ Reflective material works (n.d.). http://www.janesville.bz/3m scotchlite.html. Accessed 6 Jul 2009

4. Firefighter and fire-resistant clothing and fabric (2004) Textile J 121(3):36-42

5. ANSI/ISEA 107-2010 (2010) American national standard for high-visibility safety apparel and headwear. The International Safety Equipment Association, Arlington

6. ANSI/ISEA 207-2006 (2006) American national standard for high visibility public safety vests. The International Safety Equipment Association, Arlington

7. CSA Z96-09 (2009) High visibility safety apparel, 2nd edn. The Canadian Standards Association, Mississauga

8. DIN EN 471:2008-03 (2008) High visibility warning clothing for professional use, test methods and requirements. DIN Deutsches Institut für Normung e.V., Berlin 
9. DIN EN469:2007 (2007) Protective clothing for firefighters-performance requirements for protective clothing for firefighters. DIN Deutsches Institut für Normung e.V., Berlin

10. Tuttle SJ, Sayer JR, Buonarosa ML (2009) The conspicuity of first-responder safety garments. J Saf Res 40:191-196

11. Balk SA, Tyrrell RA, Brooks JO, Carpenter TL (2008) Highlighting human form and motion information enhances the conspicuity of pedestrians at night. Perception 37:1276-1284

12. Tyrrell RA, Wood JM, Chaparro A, Carberry TP, Chu B-S, Marsszalek RP (2009) Seeing pedestrians at night: visual clutter does not mask biological motion. Accid Anal Prev 41:506-512

13. Sayer JR, Mefford ML (2004) High visibility safety apparel and nighttime conspicuity of pedestrians in work zones. J Saf Res 35:537-546

14. Cotterill D (2009) Post-use evaluation of firefighters' turnout gear. MS thesis, University of Kentucky

15. National Fire Protection Association (2007) NFPA 1851 standard on selection, care, and maintenance of protective ensembles for structural fire fighting and proximity fire fighting 2008 edition. National Fire Protection Association, Quincy, MA, p 50

16. ASTM standard E809-08 (2008) Standard practice for measuring photometric characteristics of retroreflectors. ASTM International, West Conshohocken

17. Sayer JR, Buonarosa ML (2008) The roles of garment design and scene complexity in the daytime conspicuity of high-visibility safety apparel. J Saf Res 29:281-286

18. Luoma J, Penttinen M (1998) Effects of experience with retroreflectors on recognition of nighttime pedestrians: comparisons of driver performance in Finland and Michigan. Transp Res F 1:47-58

19. Federal Register Rules and Regulations No. 226, Friday, Nov 24 (2006) 71: 67798 